\title{
Harmonic Aggregation Techniques
}

\author{
Mohammad Mahdi Share Pasand \\ Department of Electrical and Electronics Engineering, Standard Research Institute - SRI, Alborz, Iran
}

Email address:

sharepasand@standard.ac.ir

To cite this article:

Mohammad Mahdi Share Pasand. Harmonic Aggregation Techniques. Journal of Electrical and Electronic Engineering.

Vol. 3, No. 5, 2015, pp. 117-120. doi: 10.11648/j.jeee.20150305.13

\begin{abstract}
Different harmonic aggregation techniques for assessment of current harmonic levels at points of measurement are investigated and compared to each other. The interaction effect in aggregation of harmonic currents is investigated. Several approaches including IEC 61000-3-6 recommended method are utilized and compared.
\end{abstract}

Keywords: Electromagnetic Compatibility, Harmonic Distortion, Harmonic Aggregation, Power Quality, Current Harmonics, Interaction Effect

\section{Introduction}

The importance of harmonic emission as a measurefor electromagnetic compatibility is well known. [1] Harmonic pollution, a conductive form of electromagnetic emission, may cause distribution losses, damage to several components such as transformers, power switches and electric motors, accidental operation of remotely controlled switches and breakers(false tripping), excess heating of cablings, metering errors in power distribution, penalties on monthly bill units, generator failures etc. Acceptable levels of harmonic pollution in IEC and IEEE international standards are provided as static indices measured at PCCs ${ }^{1}[3,4]$. Equipment standards determine level of current harmonic emissions from the equipment while distribution standards determine the acceptable level of voltage harmonics at the bus. In order to check whether a set of loads require harmonic filters to fulfill distribution system harmonic requirements, one has to estimate the aggregated sum of current harmonics and the voltage harmonic pollution caused by that current. Approximate methods are recommended in IEC 61000 series for evaluating the total harmonic current based on single component current [3]. The main challenge in that approximation of aggregated harmonic current is that phase behaviors of equipments are significantly different. The phase of a harmonic current depends on the equipment type (e.g. rectifier, inverter, etc.), switching/ triggering behavior (i.e. time indices at which the equipment switches on and off either by user or

1 Point of Common Coupling automatic switching mechanisms) and its parameters (e.g. threshold voltages). A significantly contributing factor is time. It is known that current pollution increases at the early night in urban and industrial areas as a result of TVs and lighting loads.

It is desirable to have an estimation of harmonic level before actually adding a new load, a harmonic filter or restructuring the network. Also aggregation is important as it makes the designer decide where to install new filters and how to distribute reactive load between different capacitive banks.

Methods for passive filter design to compensate for harmonics are surveyed in [18]. Active filters are also of growing interest as in [19]; however active filters are expensive and complicated to repair.

Street lamps and their aggregated harmonic current are studied in Aggregation of current harmonics in wind farms which is due to the time-varying behavior of wind causing varying currents and inverter voltage harmonics is studied in $[20,21]$.

Two major phenomena may impact aggregation of harmonic currents. First is the magnitude aggregation error which is caused by the phase differences between several harmonic components. Another complication is the interaction effect. Interaction is the effect of adding new loads on the harmonic components of previously installed loads. This phenomenon is caused because of line impedance which results in harmonic voltages due to harmonic currents. As a result, actual harmonic pollution of an individual load may change when installed in parallel with other loads. 


\section{Techniques for Computing Aggregated Current Harmonic}

If one sums up harmonic current magnitudes of different orders, a conservative approximation is resulted. [3] This approximated value is too large compared to actual aggregated current measured from the network. [4] In other words, actual harmonics cancels each other due to phase differences, resulting in a very smaller aggregated harmonic level than arithmetic sum. (See [6] and references therein.)

Another method is to vector-sum the harmonic currents which lead to an exact value for the aggregated value. However the actual values of harmonic phases are unknown and due to changes as a result of switching. [6] In addition, measurement of phases requires more accurate and more expensive measurement tools. An approximate method is to use statistical methods as [6] on the basis of a probability density function for harmonic phases.

The third method is to establish formulae based on empirical data. IEC 61000-3-6 recommends to add up harmonics based on table 2 and equation 2 , which is arithmetic summation for harmonic orders below 5 and a root of powered sums for higher orders.(2)

$$
\mathrm{I}_{\mathrm{hTOT}}=\sum_{\mathrm{j}=1}^{\mathrm{n}} \mathrm{I}_{\mathrm{hj}}
$$

In which the following notation is used:

$\mathrm{I}_{\text {hтот: }}$ : Aggregated harmonic current (order h) for all of components

$\mathrm{I}_{\mathrm{hj}}$ : Harmonic current (order h) for $j^{\text {th }}$ component

$$
\mathrm{I}_{\mathrm{hTOT}}=\sqrt[\alpha]{\sum_{\mathrm{j}=1}^{\mathrm{n}} \mathrm{I}_{\mathrm{hj}}{ }^{\alpha}}
$$

In which:

\begin{tabular}{|c|c|c|}
\hline & $\alpha$ & Harmonic Order \\
\hline 1 & & $\mathrm{~h}<5$ \\
\hline 1.4 & & $5<\mathrm{h}<10$ \\
\hline 2 & & $\mathrm{~h}>10$ \\
\hline
\end{tabular}

Table 1. IEC 61000-3-6 method for harmonic aggregation [5].

Other aggregation techniques include summation of vector components presuming random phase differences driven by probability densities of different types. These methods require experimental data to estimate phase angle difference PDFs, from the experimental histograms. Statistical approaches are more logical to be applied especially when an appropriate density function is available. Actual PDFs can be modeled only through complicated analytical functions. [6] In addition, because of fixed limits recommended for THD levels [4, 9], it is more practical to derive conservative deterministic models based on statistical analysis. One approach is to define a percentile in the statistical model, not to be violated by the system. For instance [10] sets 5\% limit for the THD (i.e. The THD level may not exceed $95 \%$ of the recommended limit) which is recommended by IEC as well. [3]A statistical analysis of harmonic phases is performed in [7], in which probability density functions are estimated on the basis of simplifying assumptions. The PDF of harmonic phases is considered to be uniform in [7], [6], which are too optimistic. In fact, the statistical analysis of harmonics is valid only if experimental data for individual appliance harmonics is used to model phase behaviors. In this study, experimental data are drawn to model the phase behavior, examine the current aggregation method, evaluate the statistical assumptions of [7] and to derive statistical indices for THD levels which is optimistically considered to be in steady state in standards IEEE 519, IEC 61000.

The following figures depict the approximations for aggregated harmonics. [4] RSS is suitable when phase and magnitude differences are significant. When phases are significantly different but magnitudes are the same, RSSis not appropriate. The proposed method in [4] is an appropriate choice when estimations of statistical measures of phase and magnitude are present. For identical loads with similar magnitude and phases, only linear summation is satisfactory. The practical behavior of phase and magnitudes remains as an important question which helps choosing appropriate approximation methods.

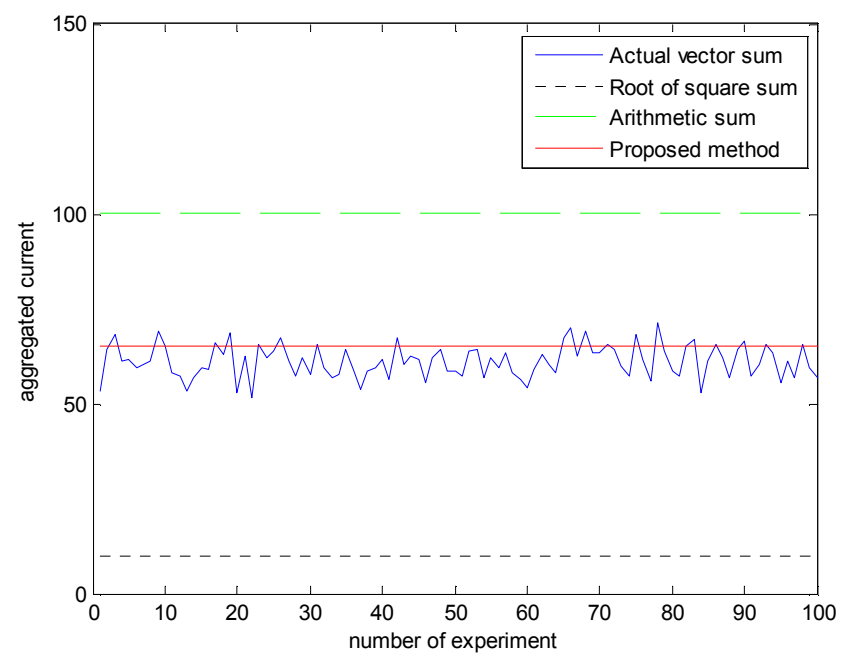

Figure 1. Comparison of different estimates: Identical loads.

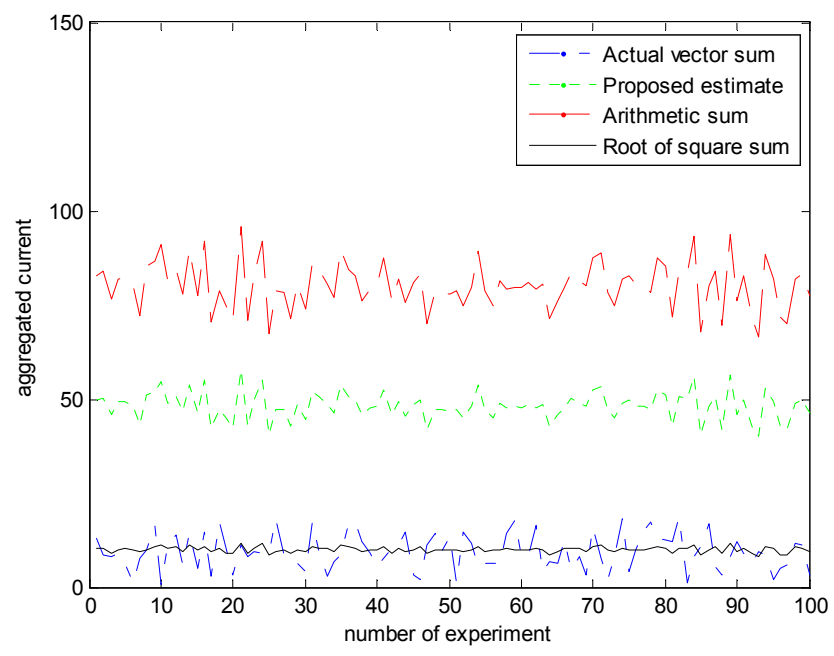

Figure 2. Comparison of different estimates: Different loads. 
It should be noted that in most applications a few number of harmonic orders are of concern. Namely $3^{\text {rd }}, 5^{\text {th }}$ and $7^{\text {th }}$ harmonics are the most important ones when current harmonic is considered. Even harmonics are usually insignificant as they usually cancel each other in the aggregation [15]. This holds also for $3^{\text {rd }}$ order harmonics in 3 phase symmetric loads. Therefore only $5^{\text {th }}$ and $3^{\text {rd }}$ harmonics are considered here to represent a measure for all harmonic orders. Existing approaches only consider aggregations of a single harmonic order and provide a total measure (e.g. THD) for all harmonics. As a result, there is no need to consider all harmonic orders in the analysis.

\section{Interaction Effects on Individual Harmonic Currents}

Interaction effect is caused because of the voltage drop between parallel loads due to resistance/impedance existing in wirings. It may also model the change in harmonic distortion due to current reduction in each load being paralleled with other loads. [22] Used simulation studies of identical and semi-identical loads of single phase rectifiers to show the effect of interaction. It is shown that interaction significantly disturbs aggregation approximations when line impedance is noticeable and harmonic currents of high orders exist in lines. This is similar to capacitive bank situation in which reactive power is preferred to be injected in lines from different places in order to reduce the distance in which reactive current is flowing.

[22] Recommends a computational method to compensate for interaction effect and improve aggregation formulae. Compared to phase effect on the aggregation, interaction is of much less effect and could be neglected unless for situations in which line impedance and THD levels are extremely high.

\section{Conclusion}

Different aggregation techniques are reviewed and the interaction effect which may adversely impact the accuracy of harmonic aggregation computations is studied. Also it is shown that phase behavior of loads has a decisive effect on the aggregated current and practical data should be gathered to extract realistic distributions for phase behavior. Effect of phase is much more severe than interaction and it could be neglected when line impedance is low.

\section{References}

[1] Allen, George W, "Design of Power-Line Monitoring Equipment", IEEE Transactions on Power Apparatus and Systems, Vol. PAS-90, Issue: 6, Nov. 1971.

[2] S Chattopadhyay, M Mitra, S Sengupta, "Electric power quality", Springer, 2011.

[3] G. D. Castro, A., M. Bollen and A. Moreno-Monuz, "Street Lamps Aggregation Analysis Through IEC 61000-3-6
Approach", International Conference on Electricity Distribution, Stockholm, June 2013.

[4] Pasand, Mohammad Mahdy Share, and Zahra Rahmatian. "Harmonic aggregation techniques for power quality assessment a standard framework." International Journal of Engineering \& Technology 3.3 (2014): 365-371.

[5] Pasand, MM Share. "Harmonic Aggregation Techniques for Power Quality Assesment A review of different methods." International Journal of Engineering Science and Technology 6.7 (2014): 423.

[6] IEEE std. 519, "Recommended Practices and Requirements for Harmonic Control in Electrical Power Systems”, 1992.

[7] IEC 61000, "Electromagnetic compatibility (EMC) - Part 3-6: Limits - Assessment of emission limits for the connection of distorting installations to MV, HV and EHV power systems", Basic EMC publication, ed.2, 2008. (IEC/TR 61000-3-6).

[8] Y. Baghzouz, et al., "Time-Varying Harmonics: Part II-Harmonic Summation and Propagation", IEEE Transactions On Power Systems, Vol. 17, No. 1, 2002.

[9] Y. Baghzouz, Task Force on Probabilistic Aspects of Harmonics "Time-varying harmonics: Part I-Characterizing measured data," IEEE Trans. Power Delivery, vol. 13, pp. 938-944, 1998.

[10] A. Cavallini, R. Langella, et al., "Gaussian modeling of harmonic vectors in power systems," in IEEE 8th Int. Conf. Harmonics and Quality of Power, Athens, Greece, Oct. 14-16, 1998, pp. 1010-1017.

[11] IEC 61000 - 3 - 2: 2009, Electromagnetic Compatibility (EMC) - Part 3-2: Limits-Limits for harmonic current emissions (Equipment input current $\leq 16 \mathrm{~A}$ per phase) (IEC 61000-3-2).

[12] CIGRE WG 36-05, "Assessing voltage quality with relation to harmonics, flicker and unbalance", France, CIGRE Rep. 36-203, $34^{\text {th }}$ Session, 1992.

[13] Medeiros, F.; Brasil, D. C.; Ribeiro, P. F.; Marques, C. A. G.; Duque, C. A. "A new approach for harmonic summation using the methodology of IEC 61400-21", $14^{\text {th }}$ International Conference on Harmonics and Quality of Power (ICHQP), 2010 .

[14] Hsiao, Y. "Design of Filters for Reducing Harmonic Distortion and Correcting Power Factor in Industrial Distribution Systems." Tamkang Journal of Science and Engineering, 4(3), 193-199, 2001.

[15] Wakileh, G. J. "Power systems harmonics: fundamentals, analysis, and filter design", Berlin: Springer, 2001.

[16] Persson, Jonas,"Comparing Harmonics Mitigation Techniques." (2014).

[17] Buccella, Concettina, et al. "Harmonic mitigation technique for multilevel inverters in power systems." Power Electronics, Electrical Drives, Automation and Motion (SPEEDAM), 2014 International Symposium on. IEEE, 2014.

[18] Aye, Thet Mon, and Soewin Naing. "Analysis of Harmonic Reduction by Using Passive Harmonic Filters." (2014).

[19] Sharma, Vandana, and Anurag Singh Tomer. "Comparative Analysis on Control Methods of Shunt Active Power Filter for Harmonics Mitigation." International Journal of Science and Research 3 (2014). 
[20] Yang, Kai, Math HJ Bollen, and E. O. Anders Larsson. "Aggregation and amplification of wind-turbine harmonic emission in a wind park." Power Delivery, IEEE Transactions on 30.2 (2015): 791-799.

[21] Yang, Kai, Math HJ Bollen, and E. O. Larsson. "Wind power harmonic aggregation of multiple turbines in power bins."
Harmonics and Quality of Power (ICHQP), 2014 IEEE 16th International Conference on. IEEE, 2014.

[22] Mohammad Mahdi Share Pasand, "Harmonic Aggregation Techniques: Methods to Compensate for Interaction Effects." American Journal of Electrical and Electronic Engineering, vol. 3, no. 3 (2015): 83-87. DOI: 10.12691/ajeee-3-3-4. 